\title{
A Systematic Approach to the Evaluation of the Influence of Multilayered Earth on Overhead Power Transmission Lines
}

\author{
Grigoris K. Papagiannis, Member, IEEE, Dimitrios A. Tsiamitros, Dimitris P. Labridis, Senior Member, IEEE, \\ and Petros S. Dokopoulos, Member, IEEE
}

\begin{abstract}
The influence of earth stratification on overhead power transmission line impedances is investigated in this paper. A systematic comparison of existing approaches is done, while results are also obtained using a finite-element method formulation. A novel numerical integration technique is proposed for the calculation of the infinite integrals involved. Typical single- and double-circuit line configurations are examined for a combination of layer depths and earth resistivities over a wide frequency range. The influence of the layer depth is also investigated. Results show significant differences from those, corresponding to the case of homogeneous earth. Using the multilayered earth return impedances in transient simulations, the transient responses show that differences occur mainly in cases of asymmetrical faults, justifying the need for a detailed earth model implementation.
\end{abstract}

Index Terms-Electromagnetic transient analysis, finite element method, nonhomogeneous earth, power transmission lines.

\section{INTRODUCTION}

$\mathbf{I}$ $\mathrm{N}$ TRANSIENT simulations, detailed transmission line modeling is required. Transmission line modeling is strongly influenced by the presence of the resistive earth return path, which is included in transmission line impedances through proper correction terms. The latter are calculated using the widely accepted Carson's formulas [1] developed for the case of semi-infinite, homogeneous earth.

In practice, earth is composed of several layers of different electromagnetic properties. Wise and Sunde [9] developed new formulas for the two-layer earth case under certain simplifications. In 1966, Wedepohl and Wasley [2] calculated the line impedances for a two-layer earth model using a double complex Fourier transform. In 1973, Nakagawa [3] proposed a more rigorous and general solution for the multilayer earth case, allowing the relative permittivity and permeability of each layer to be different from unity. Nakagawa's earth model is implemented in the electromagnetic transients program (EMTP) [11]. Finally, Moghram [4] extended in 1998 the solution of [2] for the case of a three-layer earth.

The finite element method (FEM) is a numerical method widely used for the solution of the electromagnetic field equations in a region, regardless of the geometric complexity. In a recently proposed method by some of the authors [5], the

Manuscript received May 5, 2004; revised November 11, 2004. Paper no. TPWRD-00214-2004.

The authors are with the Power Systems Laboratory, Department of Electrical and Computer Engineering, Aristotle University of Thessaloniki, GR-54124 Thessaloniki, Greece (e-mail: grigoris@eng.auth.gr).

Digital Object Identifier 10.1109/TPWRD.2005.855448

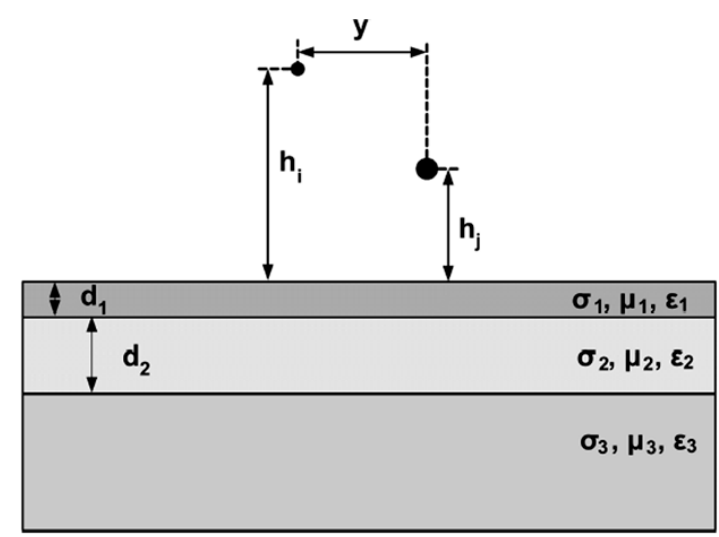

Fig. 1. Two conductors over a three-layer earth.

electromagnetic field variables have been calculated using the FEM and properly linked with the transmission line equivalent circuit parameters. The new method is capable of handling cases of terrain surface irregularities and nonhomogeneous, stratified soil, where most classical methods usually fail.

This paper presents a generalized methodology capable of handling all different approaches for the overhead transmission line impedance calculation, in cases where earth is considered to be multilayered. Instead of using infinite series approximations for the evaluation of the infinite integrals, a direct numerical integration scheme is adopted. The new method is applied to typical single- and double-circuit overhead transmission line configurations for varying soil parameters and over a wide frequency range. The obtained results show significant differences when checked against those obtained by Carson's formulas under the assumption of homogeneous earth. The validity of the results is justified using a suitable FEM formulation for the multilayered earth case. Finally the new impedances are used in typical transient simulations in order to evaluate the influence of the earth stratification on the actual voltages and currents. The resulting differences reveal that earth stratification must be taken into account in the transmission line models, when zero sequence components may occur.

\section{PROBlem Formulation}

In the general case of a two conductors $i, j$ arrangement of Fig. 1, earth is assumed to consist of three layers. The distances of the two conductors from the earth surface are $h_{i}$ and $h_{j}$ respectively, while their horizontal distance is $y$. The first earth 
layer is assumed to have a depth $d_{1}$ from the earth surface and permeability $\mu_{1}$, permittivity $\varepsilon_{1}$ and conductivity $\sigma_{1}$. The second layer has a thickness of $d_{2}$ and its electromagnetic characteristics are $\mu_{2}, \varepsilon_{2}$, and $\sigma_{2}$ respectively. The third layer is assumed to be of infinite extent, starting at a depth of $\left(d_{1}+d_{2}\right)$, with the corresponding electromagnetic characteristics being $\mu_{3}, \varepsilon_{3}$, and $\sigma_{3}$.

The influence of the imperfect earth is expressed through certain correction terms $\Delta \bar{Z}_{i i}$ and $\Delta \bar{Z}_{i j}$, which must be added to both self and mutual per-unit length conductor impedances respectively. These terms can be calculated using the following generalized expressions:

$$
\begin{aligned}
& \Delta \bar{Z}_{i i}=\frac{j \omega \mu_{0}}{\pi} \int_{0}^{\infty} e^{-2 h_{i} u} \bar{F}(u) d u \\
& \Delta \bar{Z}_{i j}=\frac{j \omega \mu_{0}}{\pi} \int_{0}^{\infty} e^{-\left(h_{i}+h_{j}\right) u} \cos (y u) \bar{F}(u) d u
\end{aligned}
$$

where $j$ is the imaginary unit, $\mu_{o}$ is the free space permeability, $\omega=2 \pi f$ is the angular velocity, $f$ is the current frequency, and $\bar{F}(u)$ is a complex function, linking the geometry of the layers with the electromagnetic properties of the earth layers.

Starting from the simplest form for the Carson's case of semiinfinite homogeneous earth, $\bar{F}(u)$ becomes more complex for the other models treating the multilayered earth cases. The analytic expressions of $\bar{F}(u)$ for each multilayered earth model are presented in Appendix I.A.

In this paper, the integral forms (1) and (2) for both homogeneous and multilayered earth cases are calculated using numerical integration rather than infinite series approximations. A combination of the Gauss-Legendre and the Gauss-Laguerre methods [14] is used for the complex integral of (1), while the Gauss-Legendre method is combined with the Lobatto rule [10] in the numerical integration of (2), due mainly to its oscillative form. This numerical integration scheme proves to be very efficient and numerically stable. The computation time for the numerical integration in all cases examined is less than $3 \mathrm{~s}$ for a set of 60 earth resistivity and frequency combinations, using an Intel Pentium IV PC at $1.7 \mathrm{GHz}$. An analytical description of the numerical integration technique is presented in Appendix I.B.

\section{FINITE ELEMENT APPROACH}

The problem of calculating the transmission line impedances can be greatly simplified, assuming that the per-unit length voltage drop $\bar{V}_{i}$ on every conductor is known for a specific current excitation. The mutual complex impedance $\bar{Z}_{i j}$ between conductor $i$ and another conductor $j$ carrying current $\bar{I}_{j}$, where all other conductors are forced to carry zero currents, is then given by

$$
\bar{Z}_{i j}=\frac{\bar{V}_{i}}{\bar{I}_{j}} \quad(i, j=1,2, \ldots, n) .
$$

The self impedance of a conductor may also be calculated from (3), by setting $i=j$. In such a case, the following procedure may be used for the calculation of the transmission line impedances [5]:
- A sinusoidal current excitation of arbitrary magnitude is applied sequentially to each conductor, while the remaining conductors are forced to carry zero currents. The corresponding voltages are recorded.

- Using (3), the self and mutual impedances of the $j$ conductor may be calculated. This procedure is repeated $n$ times, in order to calculate the impedances of $n$ conductors.

Therefore, the problem is reduced to that of calculating the actual per unit length complex voltage drops, when a current excitation is applied to the conductors. This may be achieved by a suitable FEM formulation for the solution of the linear electromagnetic diffusion equations as in [5]. Values for the current density $\bar{J}_{s z_{i}}$ on each conductor $i$ of conductivity $\sigma_{i}$ are then obtained and (3) takes the form

$$
\bar{Z}_{i j}=\frac{\bar{V}_{i}}{\bar{I}_{j}}=\frac{\bar{J}_{s z_{i}} / \sigma_{i}}{\bar{I}_{j}} \quad(i, j=1,2, \ldots, n)
$$

linking properly electromagnetic field variables and equivalent circuit parameters.

Some practical considerations concerning the application of the FEM in the problem are the following.

- The electromagnetic field of an overhead transmission line may be considered unbounded. The FEM has been used to solve unbounded field problems using several approaches. For reasons explained in [5], the extension of the discretization area (direct solution) is adopted here. The discretization area is a square $10 \mathrm{~km} \times 10 \mathrm{~km}$, with the transmission line located in its center.

- The discretization area is subdivided in first-order triangular finite elements.

- ACSR conductors are treated as tubular conductors.

- Existing symmetries in the geometry of the problem are properly utilized to improve the computational efficiency of the method.

\section{NUMERICAL RESULTS}

Each of the above methods is applied in two typical overhead line configurations, namely a single-circuit $150-\mathrm{kV}$ transmission line shown in Fig. 2(a) and a double-circuit 735-kV line with a single conductor per phase shown in Fig. 2(b).

Data for the first line were obtained by the Public Power Corporation of Greece and for the second line from [6], with all dimensions converted to SI units. The transmission line data appear in Table I.

\section{A. Two-Layer Earth Model}

First, a two-layer earth configuration is considered. Six different two-layer earth models are investigated, based on actual grounding parameter measurements [7]. The corresponding data for the layer depths and resistivities are shown in Table II. The second layer is of infinite extent. Since most soil types are nonmagnetic, the relative permeability of the earth is assumed to be equal to unity.

On the other hand, typical values for the relative earth permittivity are close to 10 . However, observing the form of $\bar{F}(u)$ for all the models [1]-[3], [9], the relative earth permittivity appears 


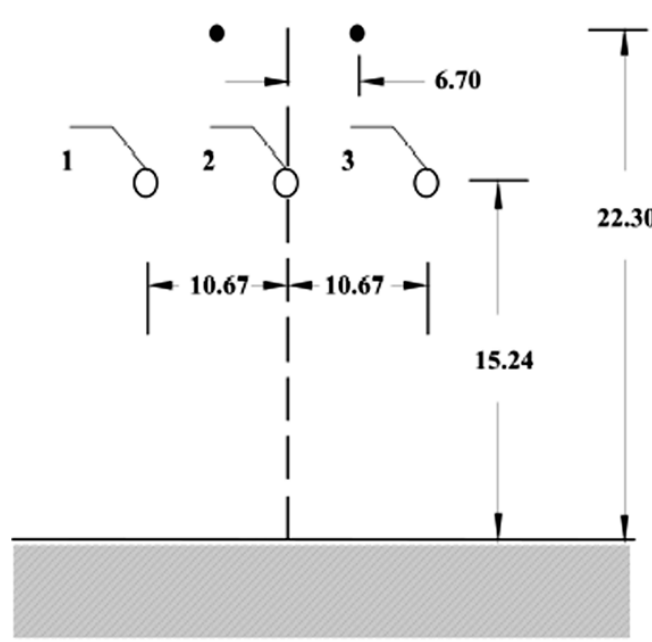

a

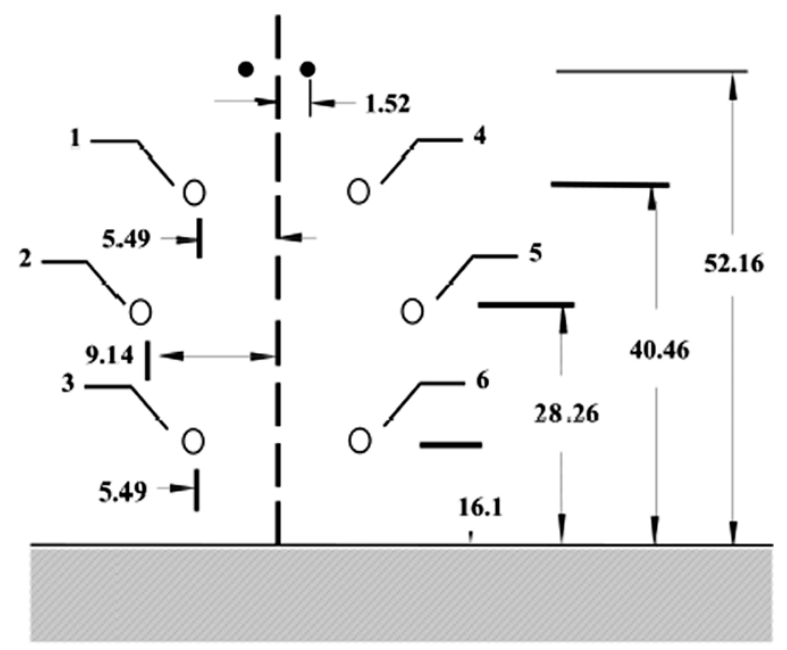

$\mathrm{b}$

Fig. 2. Transmission line configurations. (a) $150-\mathrm{kV}$ single-circuit transmission line. (b) $735-\mathrm{kV}$ double-circuit transmission line.

TABLE I

TRANSMISSION LINE DATA

\begin{tabular}{l|r|r}
\hline & $\begin{array}{c}150 \mathrm{kV} \text { single } \\
\text { circuit line } \\
\text { (Fig. 2a) }\end{array}$ & $\begin{array}{c}\text { 735kV double } \\
\text { circuit line } \\
\text { (Fig. 2b) }\end{array}$ \\
\hline Phase-conductor type & ACSR & ACSR \\
Conductor code & GROSBEAK & PHEASANT \\
Diameter (mm) & 25.146 & 35.103 \\
dc resistance $(\Omega / \mathrm{km})$ & 0.09136 & 0.04965 \\
\hline Ground-wire type & solid St & Alumoweld \\
Diameter $(\mathrm{mm})$ & conductor & Strand \\
dc resistance $(\Omega / \mathrm{km})$ & 9.525 & 9.779 \\
\hline
\end{tabular}

TABLE II

Two-LAYERED EARTH MODELS

\begin{tabular}{c|c|c|c}
\hline & $\rho_{1}(\Omega \mathrm{m})$ & $\rho_{2}(\Omega \mathrm{m})$ & $\mathrm{d}(\mathrm{m})$ \\
\hline CASE I & 372.729 & 145.259 & 2.690 \\
\hline CASE II & 246.841 & 1058.79 & 2.139 \\
\hline CASE III & 57.344 & 96.714 & 1.651 \\
\hline CASE IV & 494.883 & 93.663 & 4.370 \\
\hline CASE V & 160.776 & 34.074 & 1.848 \\
\hline CASE VI & 125.526 & 1093.08 & 2.713 \\
\hline
\end{tabular}

only in the propagation constants $\gamma_{1}$ and $\gamma_{2}$ and has a limited influence on the results for frequencies lower than $1 \mathrm{MHz}$. Thus, in all above references, the relative permittivity is considered to be equal to unity.

Frequencies from $50 \mathrm{~Hz}$ up to $1 \mathrm{MHz}$ are examined. Results are obtained by the direct numerical integration of (1) and (2) for all different approaches [2], [3], and [9]. The values of the per-cent differences between the three above approaches are calculated by

$$
\text { Difference }(\%)=\frac{\left|\bar{Z}_{\text {approach }}\right|-\left|\bar{Z}_{\text {reference }}\right|}{\left|\bar{Z}_{\text {reference }}\right|} \cdot 100 \text {. }
$$

The Wedepohl et al. [2] and Nakagawa [3] models give exactly the same results over the whole frequency range for the single- and the double-circuit lines. Keeping the Sunde approach [9] as reference, the differences between Nakagawa and Sunde are shown in Fig. 3 for the single- circuit (SC) line,

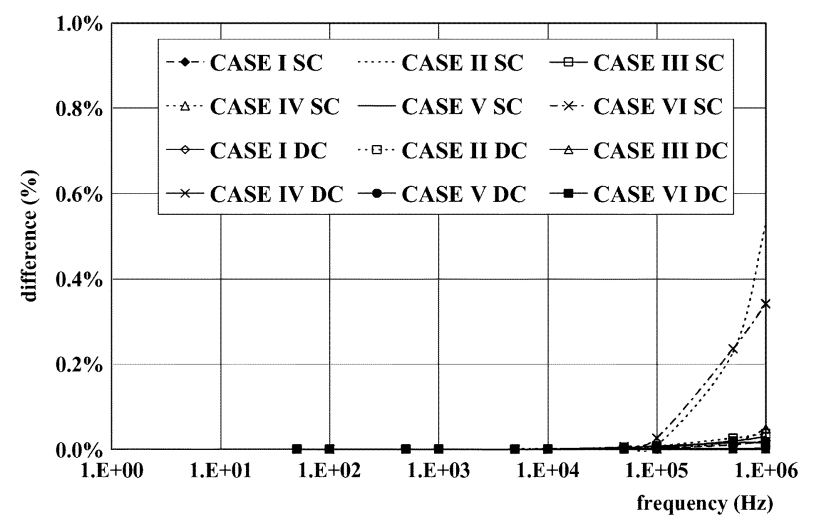

Fig. 3. Differences in the magnitude of $Z_{13}$ between Nakagawa and Sunde models for the case of the SC line and between Wedepohl and Sunde models for the double-circuit line.

TABLE III

THREe-LAYERED EARTH MODEl DATA

\begin{tabular}{c|c|c|c|c|c}
\hline & $\rho_{1}(\Omega \mathrm{m})$ & $\rho_{2}(\Omega \mathrm{m})$ & $\rho_{3}(\Omega \mathrm{m})$ & $\mathrm{d}_{1}(\mathrm{~m})$ & $\mathrm{d}_{2}(\mathrm{~m})$ \\
\hline CASE I & 30 & 9.4 & 500 & 3.4 & 25.5 \\
\hline CASE II & 128 & 1930 & 520 & 3.1 & 15 \\
\hline
\end{tabular}

as well as the differences between Wedepohl and Sunde for the double-circuit line. The differences are almost zero except for the high frequency range, where they are less than $0.6 \%$.

\section{B. Three-Layer Earth Model}

Next, the case of a three-layer earth model is considered. Two different three-layer earth models are investigated, based on actual grounding parameter measurements [8]. The corresponding data for the layers geometry and resistivities are shown in Table III. The third layer is of infinite extent. For the reasons explained earlier, the relative permeability and permittivity of the three layers is considered to be equal to unity.

Frequencies from $50 \mathrm{~Hz}$ up to $1 \mathrm{MHz}$ are examined. Results are obtained by Nakagawa [3] and Moghram [4] models. Both approaches give exactly the same results for the whole frequency range and for the single- and the double-circuit lines. 


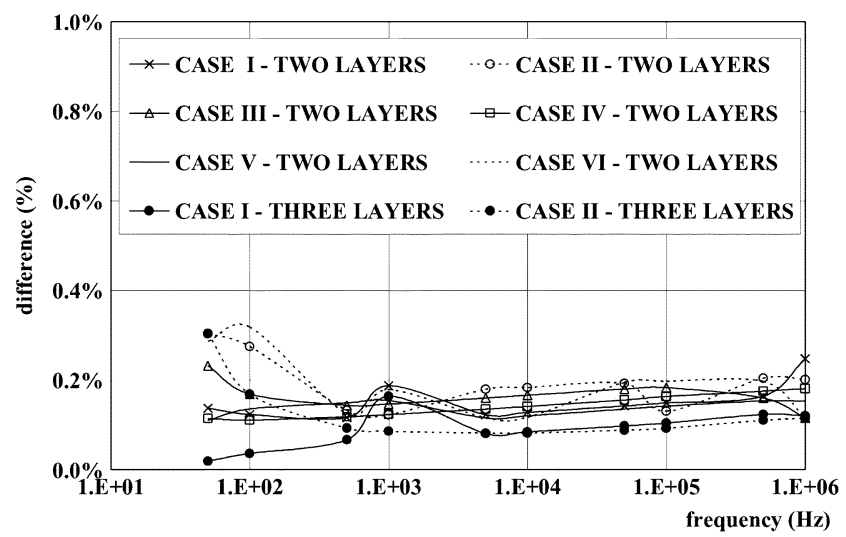

Fig. 4. Differences in $Z_{13}$ between Wedepohl and the FEM for the two-layer earth and the double-circuit line and between Nakagawa and the FEM for the three-layer earth and the single- circuit line.

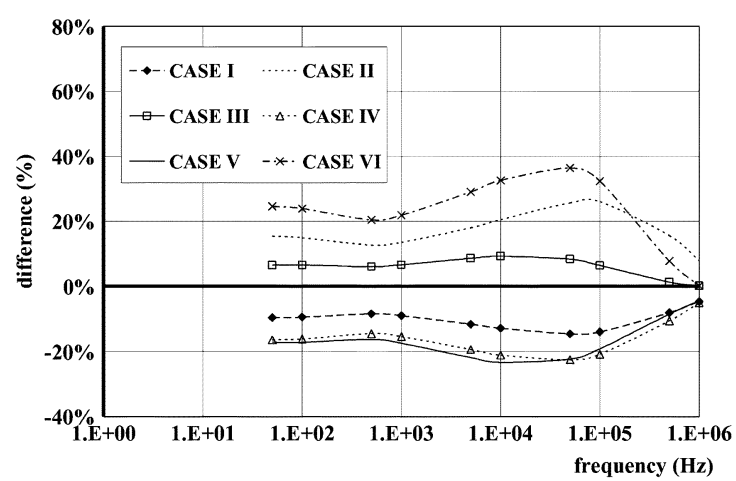

Fig. 5. Differences in the magnitude of $Z_{13}$, for the case of the single- circuit line between the homogeneous and the two-layer earth models.

\section{Comparison With the FEM and the Homogeneous Earth}

All the approaches for the two- and the three-layer earth cases are compared to the FEM formulation for the singleand the double-circuit lines. The differences given by (5), with the FEM results as reference, are calculated. The differences between Wedepohl [2] and the FEM for the two-layer earth and the double-circuit line, as well as between Nakagawa [3] and the FEM for the three-layer earth and the single- circuit line are shown in Fig. 4, for the magnitude of the mutual impedance.

In all examined cases, the recorded differences are less than $0.3 \%$ over the whole frequency range. Therefore, the validity of all approaches for both two- and three-layer earth structures is justified.

The two-layer earth impedances are also compared to the corresponding results for the homogeneous earth with resistivity equal to the first earth layer. The differences are calculated by (5), considering the homogeneous earth results as reference. In Fig. 5 the differences for the magnitude of the single-circuit transmission line mutual impedances reach almost 38\%, due to the two-layer earth stratification. The worst case is case VI, where the divergence between the resistivities of the first and the second earth layer is the highest. Differences reach a maximum at the kilohertz frequency range, affecting mainly the switching transient modeling.

For the case of the double-circuit line and the two-layer earth, the differences in the magnitude of the transmission line self impedances are presented in Fig. 6. Results for one case with no

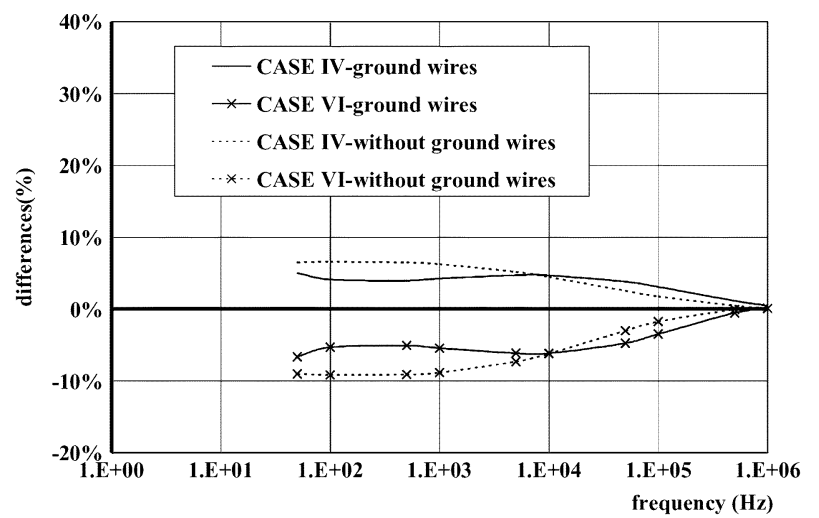

Fig. 6. Differences in the magnitude of $Z_{33}$, for the case of the double-circuit line between the homogeneous and the two-layer earth models.

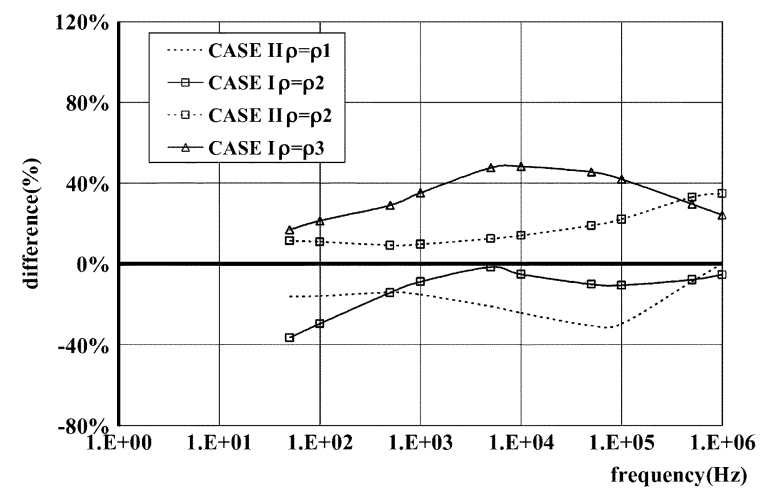

Fig. 7. Differences in the magnitude of $Z_{23}$, for the case of the single- circuit line between the homogeneous and the three-layer earth models.

ground wires are also presented to show that the ground wires have a smoothing effect in the recorded differences as they provide a parallel path for the earth return currents.

In Figs. 5 and 6, it is obvious that the differences seem to minimize near high frequencies, for which the penetration depth $p$ [13], given by (6), approaches the depth of the first layer.

$$
p=\frac{1}{\sqrt{\pi f \mu \sigma}}
$$

The results by the three-layer earth models are compared to those obtained by the Carson model for the case of semi-infinite homogeneous earth with a resistivity successively set equal to the resistivity of each of the three earth layers. The percent differences calculated by (5), using the homogeneous earth as reference, are plotted in Fig. 7 for the mutual impedance of the single- circuit line.

Significant differences of up to $50 \%$ are recorded once again for most cases. These differences tend to minimize when the resistivity of the homogeneous earth is selected to be equal to the layer that according to the penetration depth, has the more pronounced influence on the transmission line electromagnetic field.

The numerical integration scheme proved to be numerically stable and efficient in all cases examined. The results obtained by the numerical integration show a very good agreement to those obtained by the FEM for all multilayered earth models in all cases examined. Therefore, the method can be implemented as a general purpose numerical evaluation tool for all 


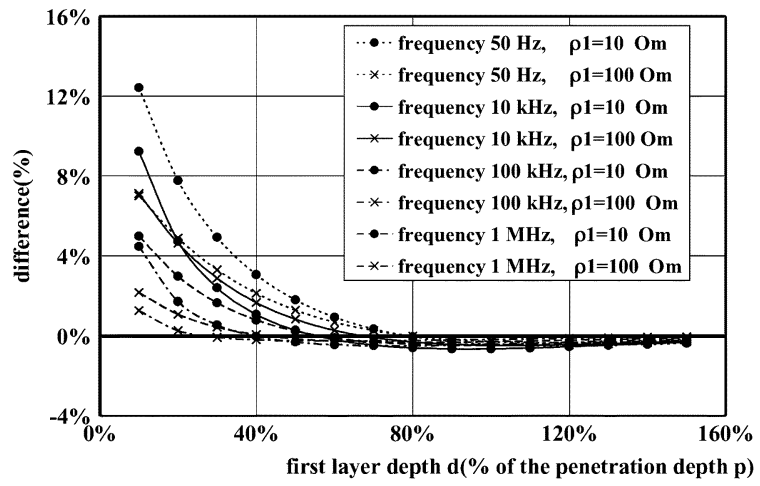

Fig. 8. Influence of the earth layer thickness. Differences in the magnitude of $Z_{11}$, for the single-circuit line between the homogeneous and the two-layer earth models.

available multilayered earth models without the numerical assumptions associated with the calculation procedure for each different model.

The influence of the thickness of the earth layers is examined next. For frequencies of $50 \mathrm{~Hz}, 10 \mathrm{kHz}, 100 \mathrm{kHz}$, and $1 \mathrm{MHz}$, the first earth layer, in a two-layer configuration, is considered to have a depth $d$ varying from $10 \%-150 \%$ of the corresponding penetration depth $p$ calculated by (6). Earth resistivities of 10 $\Omega \mathrm{m}$ and $100 \Omega \mathrm{m}$ for the first layer are examined, while the resistivity of the second layer is always $1000 \Omega \mathrm{m}$. The results of this analysis, for the differences in the magnitude of the self impedance for the single- circuit line, calculated by (5), are presented in Fig. 8.

It is shown that in cases where the resistivity of the first layer differs greatly from the second, the influence of the earth stratification is maximized. The next remarkable point is that the differences are minimized when the depth of the first earth layer reaches the $60 \%-70 \%$ of the penetration depth $p$. For high frequencies close to $1 \mathrm{MHz}$, the differences are minimized, because of the very small penetration depth $p$ at this frequency region. For these high frequencies, earth may be considered to be homogeneous having the properties of the first earth layer. Therefore, in the case of overhead lines, the use of the penetration depth may prove to be an easy and practical tool for approximation when neglecting earth stratification.

\section{Influence of Earth Stratification on Transient Response}

Of vital importance is the investigation of the influence of earth stratification on the actual transient voltages and currents resulting from a transient simulation where the line models take into account the multilayered earth. To demonstrate the effect of ignoring soil stratification, the results obtained by the numerical integration method for a two- and a three-layer earth case are used in a switching transient simulation. The BPA field test configuration, described in [12], is adapted as shown in Fig. 9. The transmission line arrangement of Fig. 2(a) is considered with a total length of $200 \mathrm{~km}$. Three different types of transients are examined. A 3-phase to ground fault at the receiving end LM through $2 \Omega$ fault resistances, a single line to ground (SLG) fault through a $2 \Omega$ resistance at the receiving end phase $c$ and an open-ended line energization through the switches at the sending end JD. All switching actions occur at $t=10.15 \mathrm{~ms}$.
A time domain transmission line model is used with distributed parameters obtained by the EMTP LINE CONSTANTS supporting routine [11], for all the cases of Tables II and III, considering the semi-infinite homogeneous earth to have a resistivity equal to the corresponding first layer. The same model is once again used but with its parameters calculated by the numerical integration method for the same cases, taking into account the earth stratification.

In both cases, all the line parameters are calculated at 500 Hz. Fig. 10(a) shows the voltage of the phase $b$ receiving end for the SLG fault. The results by the three-layered earth CASE I model are compared to those by the homogeneous earth model. In Fig. 10(b), the fault current for the same transient and for the CASE VI of the two-layer earth, is superimposed on the corresponding curve for the homogeneous earth. Finally, in Fig. 10(c), the phase $b$ open end voltage of the energization transient for the CASE II of the three-layer earth is superimposed on the corresponding curve for the homogeneous earth. The results for the fault currents in the case of the 3-phase to ground fault are practically identical for the homogeneous and the stratified earth cases.

The results by the transient simulations show that the earth stratification has negligible effect in cases of symmetrical faults and transients. In cases of asymmetrical faults, differences occur in the voltages and currents, which are generally lower than the differences in the impedances of the lines, but significant enough to be disregarded.

The reason for this is that in the case of asymmetrical transients the residual currents flow through the earth, while in the symmetrical transients these currents are minimized. The differences in the mutual and self impedances, due to earth stratification, are subtracted in the line modes and are accumulated in the ground mode. Clearly, earth stratification proves to be significant for the ground mode but minimal for the line modes [15].

In the SLG fault case, the largest differences occur once again for the cases, where substantial differences exist in the layer resistivities as well as in the cases where the overall geometry shows major divergence from the semi-infinite earth case. In Fig. 10(a), the nonhomogeneous earth case shows the worst transient while the opposite situation is recorded for the fault current in Fig. 10(b).

It has been also observed that the two-layer earth model gives the worst transient, when the resistivity of first layer is greater than the corresponding of the second layer. In the opposite case, the worst transient occurs for the homogeneous earth model.

The recorded maximum differences in peak to peak transient voltages and currents for all examined cases indicate the quantitative influence of the earth stratification and are presented as absolute values in Table IV. All results are for a 200-km-long single-circuit line.

\section{CONCLUSION}

A generalized methodology for the evaluation of the influence of earth stratification on the impedances of actual overhead transmission lines configurations is presented in this paper. The methodology can be applied to all available earth modeling approaches. 


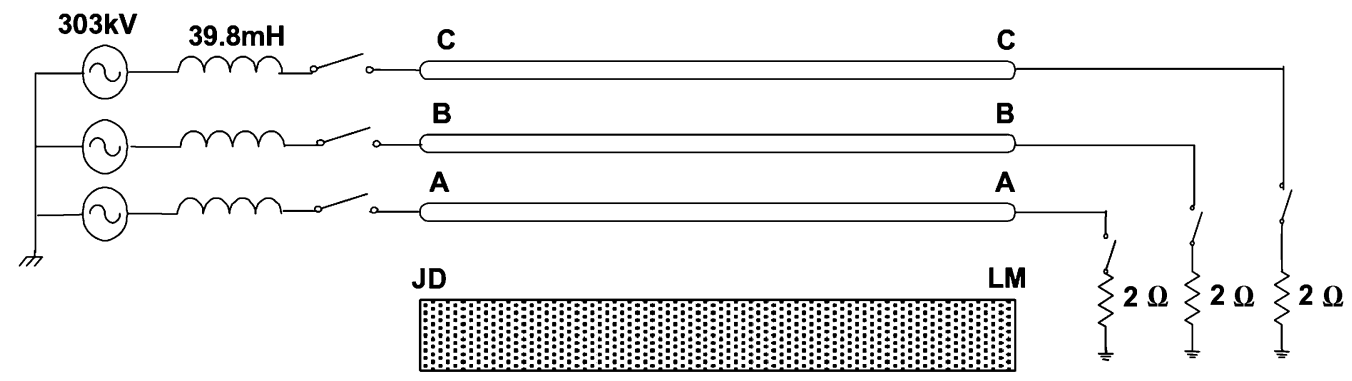

Fig. 9. Test configuration for switching transient simulation.

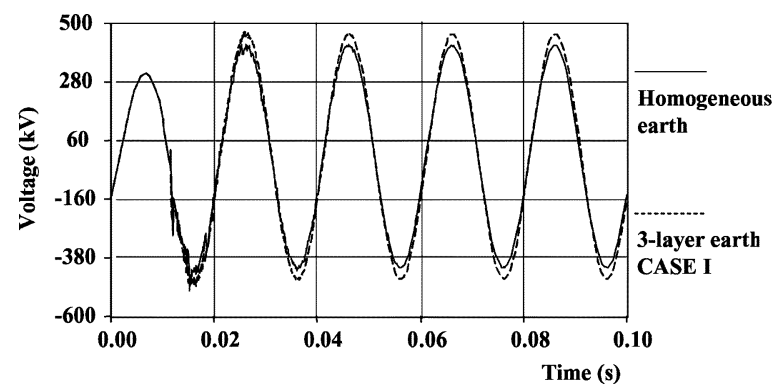

(a)

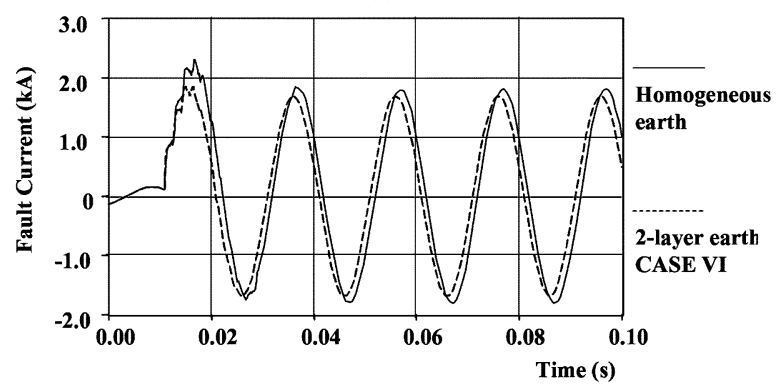

(b)

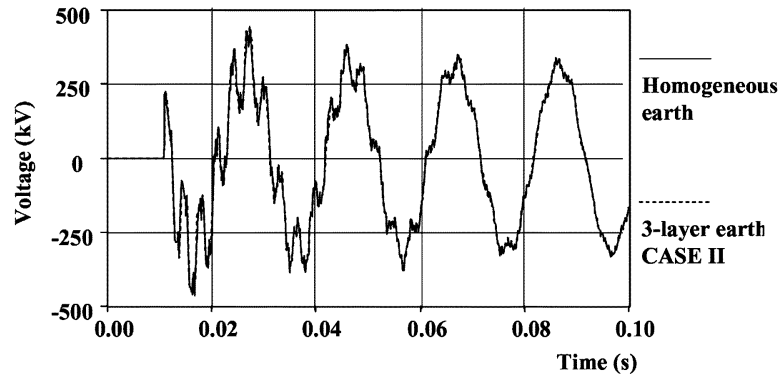

(c)

Fig. 10. (a) SLG fault open phase $b$ voltage. (b) SLG fault current. (c) Line energization phase $b$ receiving end voltage.

A novel direct numerical integration scheme is adopted for the calculation of the infinite integrals. The proposed scheme proves to be numerically stable and efficient and can be used in all cases, eliminating the need for model specific assumptions in the numerical evaluation process. The accuracy of the results obtained using numerical integration is verified by comparison with the corresponding results obtained by a recently proposed FEM formulation, suitable for the calculation of transmission line impedances.

Typical single- and double-circuit overhead transmission line configurations are analyzed. Several combinations of layer depths and layer resistivities, based on experimental data, are considered in a frequency range from $50 \mathrm{~Hz}$ to $1 \mathrm{MHz}$. Results
TABLE IV

PEAK to Peak Relative (\%) DifFerEnCES BetweEn HomogeneOUS AND MulTiLAYERED EARTH MODELS

\begin{tabular}{l|l||c||c|c||c}
\hline \hline & & $\begin{array}{c}3 \text { PHASE } \\
\text { FAULT } \\
\text { CURRENT }\end{array}$ & $\begin{array}{c}\text { SLG } \\
\text { FAULT } \\
\text { CURRENT }\end{array}$ & $\begin{array}{c}\text { SLG } \\
\text { FAULT } \\
\text { PHASE b } \\
\text { VOLTAGE }\end{array}$ & $\begin{array}{c}\text { LINE } \\
\text { ENERG. } \\
\text { PHASE b } \\
\text { VOLTAGE }\end{array}$ \\
\hline \multirow{2}{*}{$\begin{array}{l}\text { layer } \\
\text { earth }\end{array}$} & CASE I & $3.71 \%$ & $8.13 \%$ & $2.93 \%$ & $0.16 \%$ \\
\cline { 2 - 6 } & CASE II & $0.84 \%$ & $12.64 \%$ & $5.31 \%$ & $0.01 \%$ \\
\cline { 2 - 6 } & CASE III & $0.72 \%$ & $5.11 \%$ & $2.72 \%$ & $0.29 \%$ \\
\cline { 2 - 6 } & CASE IV & $2.10 \%$ & $15.23 \%$ & $5.50 \%$ & $0.19 \%$ \\
\cline { 2 - 6 } & CASE V & $3.75 \%$ & $15.71 \%$ & $6.39 \%$ & $0.32 \%$ \\
\cline { 2 - 6 } & CASE VI & $1.01 \%$ & $18.08 \%$ & $7.49 \%$ & $0.18 \%$ \\
\hline \hline \multirow{2}{*}{$\begin{array}{l}\text { layer } \\
\text { earth }\end{array}$} & CASE I & $4.05 \%$ & $9.50 \%$ & $5.26 \%$ & $0.47 \%$ \\
\cline { 2 - 6 } & CASE II & $3.81 \%$ & $11.82 \%$ & $4.68 \%$ & $0.17 \%$ \\
\hline \hline
\end{tabular}

obtained by all the above approaches are compared to the corresponding results for semi-infinite homogeneous earth.

The analysis shows that all approaches give almost identical results. Significant differences to the homogeneous earth case are recorded for the self and mutual line impedances, due to earth stratification. These differences range from $10 \%$ up to $55 \%$ depending on the layer configuration and properties. The higher differences occur in the kilohertz frequency range.

The penetration depth can be used as a practical method to choose a proper homogeneous earth model and as a rough estimate of the introduced approximation.

Finally the influence of earth stratification on the transient response of a transmission line is checked. Simple switching transients involving line energization, single line and 3-phase to ground faults are examined. Results show that the earth stratification has a minimal influence on the transient response in cases of symmetrical transients. In cases of asymmetries, where the ground mode is dominant, the influence of the earth stratification is significant enough to be discarded. The implementation of the proposed approach may offer an efficient evaluation tool in such cases.

\section{APPENDIX}

\section{A. Formulas}

Equations (1) and (2) give a generalized form for the earth correction terms for self and mutual per-unit length impedances. Assuming that $\mu=\varepsilon=1$ and $\sigma_{0}=0$ above the earth for all cases, the complex function $\bar{F}(u)$ may be expressed as follows for the different earth models. 
1) Carson's Semi-Infinite Homogeneous Earth [1]:

$$
\bar{F}(u)=\frac{1}{u+\sqrt{u^{2}+j \omega \mu_{0} \sigma}}
$$

where $\sigma$ is the earth conductivity in $\mathrm{S} / \mathrm{m}, \mu_{0}$ the permeability of the free space and $\omega=2 \pi f$ the angular velocity in $\mathrm{s}^{-1}$.

2) Two-Layer Earth Model by Sunde [9]:

$$
\bar{F}(u)=\frac{\alpha_{1}+\alpha_{2}+\left(\alpha_{1}-\alpha_{2}\right) e^{-2 \alpha_{1} d_{1}}}{\left(\alpha_{1}+\alpha_{2}\right)\left(u+\alpha_{1}\right)+\left(\alpha_{1}-\alpha_{2}\right)\left(u-\alpha_{1}\right) e^{-2 \alpha_{1} d_{1}}}
$$

where

$$
\begin{aligned}
& \alpha_{1}=\sqrt{u^{2}+\gamma_{1}^{2}}, \quad \text { and } \quad \gamma_{1}^{2}=j \omega \mu_{1} \sigma_{1} \\
& \alpha_{2}=\sqrt{u^{2}+\gamma_{2}^{2}}, \quad \text { and } \quad \gamma_{2}^{2}=j \omega \mu_{2} \sigma_{2}
\end{aligned}
$$

and $d_{1}$ is the thickness of the first earth layer, as shown in Fig. 1.

3) Two-Layer Earth Model by Wedepohl-Wasley [2]:

$$
\begin{aligned}
\bar{F}(u)= & \frac{1}{u+\left(\mu_{0} / \mu_{1}\right) \bar{A}(u)} \\
\bar{A}(u)= & \frac{c_{1}}{\sinh \left(d_{1} c_{1}\right)}\left(\cosh \left(d_{1} c_{1}\right)\right. \\
& \left.-\frac{c_{1}}{c_{1} \cosh \left(d_{1} c_{1}\right)+\left(\mu_{1} / \mu_{2}\right) c_{2} \sinh \left(d_{1} c_{1}\right)}\right)
\end{aligned}
$$

where

$$
c_{1}=\sqrt{u^{2}+j \omega \mu_{1} \sigma_{1}} \text { and } c_{2}=\sqrt{u^{2}+j \omega \mu_{2} \sigma_{2}}
$$

and $d_{1}$ is the thickness of the first earth layer, as shown in Fig. 1.

4) Three-Layer Earth Model Extension by Moghram [4]: In this extension, the expression for $\bar{F}(u)$ is similar to (A6). Equation (A7) is modified to include the third earth layer as follows:

$$
\begin{aligned}
\bar{A}(u) & =\frac{c_{1}}{\sinh \left(d_{1} c_{1}\right)}\left(\cosh \left(d_{1} c_{1}\right)\right. \\
& \left.-\frac{c_{1}}{c_{1} \cosh \left(d_{1} c_{1}\right)+\left(\mu_{1} / \mu_{2}\right) c_{2} \sinh \left(d_{1} c_{1}\right) \bar{K}(u)}\right)
\end{aligned}
$$

where

$$
\bar{K}(u)=\frac{\left(\mu_{2} / \mu_{3}\right) c_{3} \cosh \left(d_{2} c_{2}\right)+c_{2} \sinh \left(d_{2} c_{2}\right)}{c_{2} \cosh \left(d_{2} c_{2}\right)+\left(\mu_{2} / \mu_{3}\right) c_{3} \sinh \left(d_{2} c_{2}\right)}
$$

and

$$
\begin{aligned}
& c_{1}=\sqrt{u^{2}+j \omega \mu_{1} \sigma_{1}}, \quad c_{2}=\sqrt{u^{2}+j \omega \mu_{2} \sigma_{2}} \\
& c_{3}=\sqrt{u^{2}+j \omega \mu_{3} \sigma_{3}} .
\end{aligned}
$$

5) N-Layer Earth Model by Nakagawa [3]: Although it was used only for the cases of two and three-layer earth, this model is capable of handling the $N$-layer earth case

$$
\bar{F}(u)=\frac{\bar{F}_{1}(u)+\bar{G}_{1}(u)}{\left(u+\mu_{0} b_{1}\right) \bar{F}_{1}(u)+\left(u-\mu_{0} b_{1}\right) \bar{G}_{1}(u)} .
$$

Complex functions $\bar{F}_{1}(u)$ and $\bar{G}_{1}(u)$ are calculated by the following recursive formulas:

$$
\begin{aligned}
& \bar{F}_{N-1}(u)= b_{N-1}+b_{N} \\
& \bar{G}_{N-1}(u)=\left(b_{N-1}-b_{N}\right) e^{-2 \alpha_{N-1} t_{N-1}} \\
& \vdots \\
& \bar{F}_{m}(u)=\left(b_{m}+b_{m+1}\right) \bar{F}_{m+1}(u) \\
&+\left(b_{m}-b_{m+1}\right) \bar{G}_{m+1}(u) e^{2 \alpha_{m+1} t_{m}} \\
& \bar{G}_{m}(u)= {\left[\left(b_{m}-b_{m+1}\right) \bar{F}_{m+1}(u)\right.} \\
&\left.+\left(b_{m}+b_{m+1}\right) \bar{G}_{m+1}(u) e^{2 \alpha_{m+1} t_{m}}\right] e^{-2 \alpha_{m} t_{m}} \\
& \quad(1 \leq m \leq N-2) . \quad(\mathrm{A} 12)
\end{aligned}
$$

In (A12), $N$ is the number of earth layers, $t_{m}$ the distance of the lower boundary surface of the $m$ layer from the surface of the earth. The relation between $t$ and $d$ is given by

$$
\begin{aligned}
t_{1} & =d_{1} \\
t_{m} & =\sum_{1}^{m} d_{i} .
\end{aligned}
$$

The rest of the variables are given by the following equations:

$$
\begin{aligned}
& \alpha_{i}=\sqrt{u^{2}+k_{0}^{2}-k_{i}^{2}}, \quad b_{i}=a_{i} / \mu_{i} \quad(i=1,2, \ldots, N) \\
& k_{i}^{2}=-j \omega \mu_{i}\left(\sigma_{i}+j \omega \varepsilon_{i}\right), \quad k_{0}^{2}=-j \omega \mu_{0}\left(\sigma_{0}+j \omega \varepsilon_{0}\right)
\end{aligned}
$$

where $\varepsilon_{0}$ is the free space permittivity.

\section{B. Numerical Integration Method}

A novel numerical integration technique is applied for the calculation of the infinite integrals of (1) and (2). It is based on the presence of the terms $e^{-\left(h_{i}+h_{j}\right) u}$ and $e^{-2 h_{i} u}$ in the integrand and on the observation that the integral of (2) has as a factor the term $\cos (u y)$. Therefore, some of the roots of the integrand are known. Thus, when the horizontal distance $y$ between the two conductors is not zero, the integral in the right part of (2) can be calculated as follows.

The Gauss-Legendre method [14] is applied to calculate the integral between zero and the first root of $\cos (u y)$, as this method is capable of handling the initial steep descent of the integrand. Then the Lobatto rule [10] is applied in the intervals between subsequent roots of $\cos (u y)$. The procedure is repeated until convergence is achieved, controlled by a user defined tolerance, set to $10^{-9}$ in this paper.

For the calculation of the integral in (1) or the integral in (2) in the case where the horizontal distance $y$ is zero, the procedure is described below.

The Gauss-Legendre method is applied in order to find the integral at an initial interval between zero and $u_{0}=4 \times 10^{-4} /\left(h_{i}+h_{j}\right)$ in (2) or $u_{0}=4 \times 10^{-4} / 2 h_{i}$ in (1). Then the shifted Gauss-Laguerre method [14] is used for the evaluation of the rest of the integral. The procedure is repeated iteratively. In each iteration, the initial interval is bisected and 
the use of the Gauss-Legendre method is extended by $10 u_{0}$ intervals to the right of $u_{0}$. Convergence is achieved when the absolute difference between two succedent values of calculated integral is less than the predefined tolerance.

In all cases examined, convergence is achieved after $3-4$ iterations.

\section{REFERENCES}

[1] J. R. Carson, "Wave propagation in overhead wires with ground return," Bell Syst. Tech. J., no. 5, pp. 539-554, 1926.

[2] L. M. Wedepohl and R. G. Wasley, "Wave propagation in multiconductor overhead lines-Calculation of series impedance for multilayer earth," Proc. IEE, vol. 113, no. 4, pp. 627-632, 1966.

[3] M. Nakagawa, A. Ametani, and K. Iwamoto, "Further studies on wave propagation in overhead transmission lines with earth return: Impedance of stratified earth," Proc. IEE, vol. 120, no. 12, pp. 1521-1528, 1973.

[4] I. S. Moghram, "Effects of earth stratification on impedances of power transmission lines," Eur. Trans. Elect. Power (ETEP), vol. 8, no. 6, pp. 445-449, 1998.

[5] G. K. Papagiannis, D. G. Triantafyllidis, and D. P. Labridis, "A one-step finite element formulation for the modeling of single and double circuit transmission lines," IEEE Trans. Power Syst., vol. 15, no. 1, pp. 33-38, 2000.

[6] P. C. Magnusson, "Travelling waves on multi-conductor open-wire lines: A numerical survey of the effects of frequency dependence on modal decomposition," IEEE Trans. Power App. Syst., vol. PAS-92, no. 3, pp. 999-1008, 1973.

[7] J. L. del Alamo, "A comparison among different techniques to achieve an optimum estimation of electrical grounding parameters in two-layered earth," IEEE Trans. Power Del., vol. PWRD-8, no. 4, pp. 1890-1899, 1993

[8] P. J. Lagace, J. Fortin, and E. D. Crainic, "Interpretation of resistivity sounding measurements in N-layer soil using electrostatic images," IEEE Trans. Power Del., vol. 11, no. 3, pp. 1349-1354, 1996.

[9] E. D. Sunde, Earth Conduction Effects in Transmission Systems, 2nd ed. New York: Dover, 1968, pp. 99-139.

[10] P. Davies and P. Rabinowitch, Methods of Numerical Integration, 2nd ed. New York: Academic, 1984, p. 104, 223.

[11] H. W. Dommel, "Electromagnetic transients program reference manual," Portland, OR: Bonneville Power Administration, 1986, pp. 4-4.

[12] W. S. Meyer and H. W. Dommel, "Numerical modeling of frequency-dependent transmission line parameters in an electromagnetic transients program," IEEE Trans. Power App. Syst., vol. PAS-93, no. 5, pp. $1401-1409,1974$.

[13] P. C. Magnusson, G. C. Alexander, and V. K. Tripathi, Transmission Lines and Wave Propagation, 3rd ed. Boca Raton, FL: CRC Press, 1992, p. 229.

[14] K. E. Atkinson, An Introduction to Numerical Analysis, 2nd ed. New York: Wiley, 1987, pp. 270-282, 308-309.

[15] A. Semlyen, "Accuracy limits in the computed transients on overhead lines due to inaccurate ground return modeling," IEEE Trans. Power Del., vol. PWRD-17, no. 3, pp. 872-878, Jul. 2002.
Grigoris K. Papagiannis (S'79-M'88) was born in Thessaloniki, Greece, in 1956. He received the Dipl.-Eng. and Ph.D. degrees from the Department of Electrical Engineering, Aristotle University of Thessaloniki, Thessaloniki, Greece, in 1979 and 1998, respectively.

Since 1981, he has been a Research Assistant and since 1998 a Lecturer at the Power Systems Laboratory, Department of Electrical and Computer Engineering, Aristotle University of Thessaloniki. His special interests are power system analysis, with emphasis on modeling and computation of electromagnetic transients and powerline communications.

Dimitrios A. Tsiamitros was born in Kozani, Greece, in 1979. He received the Dipl.-Eng. degree from the Department of Electrical and Computer Engineering, Aristotle University of Thessaloniki, Thessaloniki, Greece, in 2001.

Since 2001, he has been a postgraduate student at the Department of Electrical and Computer Engineering, Aristotle University of Thessaloniki. His special interests are power system modeling and computation of electromagnetic transients.

Dimitris P. Labridis (S'88-M'90-SM'00) was born in Thessaloniki, Greece, on July 26, 1958. He received the Dipl.-Eng. and Ph.D. degrees from the Department of Electrical Engineering, Aristotle University of Thessaloniki, in 1981 and 1989, respectively.

During 1982-2001, he was a Research Assistant and later a Lecturer and Assistant Professor at the Department of Electrical Engineering, Aristotle University of Thessaloniki. Since 2001, he has been an Associate Professor in the same department. His special interests are power system analysis with special emphasis on the simulation of transmission and distribution systems, electromagnetic and thermal field analysis, numerical methods in engineering, artificial intelligence applications in power systems, and powerline communications.

Petros S. Dokopoulos (M'77) was born in Athens, Greece, in 1939. He received the Dipl.Eng. degree from the Technical University of Athens, Athens, Greece, in 1962 and the Ph.D. degree from the University of Brunswick, Brunswick, Germany, in 1967.

During 1962-1967, he was with the Laboratory for High Voltage and Transmission, University of Brunswick, during 1967-1974 with the Nuclear Research Center, Julich, Germany, and during 1974-1978 with the Joint European Torus. Since 1978, he has been a Full Professor at the Department of Electrical Engineering at the Aristotle University of Thessaloniki, Thessaloniki, Greece. He has worked as a Consultant to Brown Boveri and Cie, Mannheim, Germany, to Siemens, Erlagen, Germany, to Public Power Corporation, Greece, and to National Telecommunication Organization and construction companies in Greece. His scientific fields of interest are dielectrics, power switches, power generation (conventional and fusion), transmission, distribution, and control in power systems. He has 88 publications and seven patents on these subjects. 